\title{
Embedding odometers in cellular automata
}

\author{
by \\ Ethan M. Coven (Middletown, CT) and \\ Reem Yassawi (Peterborough, ON)
}

To Michat Misiurewicz with admiration and affection

\begin{abstract}
We consider the problem of embedding odometers in one-dimensional cellular automata. We show that (1) every odometer can be embedded in a gliders-withreflecting-walls cellular automaton, which one depending on the odometer, and (2) an odometer can be embedded in a cellular automaton with local rule $x_{i} \mapsto x_{i}+x_{i+1}$ mod $n$ $(i \in \mathbb{Z})$, where $n$ depends on the odometer, if and only if it is "finitary."
\end{abstract}

1. Introduction. An odometer is the "+1" map on a countable product of finite cyclic groups. A (one-dimensional) cellular automaton $(X, T)$ is a dynamical system defined by a local rule on a closed, $T$-invariant subset of either $A^{\mathbb{N}}$ or $A^{\mathbb{Z}}$, where $A$ is a finite alphabet. In [3] the authors and M. Pivato partially solved the "give me a cellular automaton and I will find an odometer that can be embedded in it" problem. In this paper we completely solve the converse problem: "give me an odometer and I will find a cellular automaton that it can be embedded in."

THEOREM 1. Every odometer can be embedded in a gliders-with-reflecting-walls cellular automaton.

Although finitary odometers (defined in Theorem 2 below) can be embedded in a number of cellular automata [7], Theorem 1 identifies a (relatively small) class of cellular automata such that every odometer can be embedded in one of them.

THEOREM 2. Every finitary odometer $(\mathbb{Z}(S),+1)$, i.e. one such that the set of prime divisors of the members of $S$ is finite, can be embedded in the one-dimensional, two-sided cellular automaton with local rule $x_{i} \mapsto x_{i}+$ $x_{i+1} \bmod n(i \in \mathbb{Z})$, defined on the space of all doubly infinite sequences with 
entries from $\mathbb{Z}_{n}$, the ring of integers modulo $n$, where $n$ is the product of the primes that divide infinitely members of $S$.

Conversely, only finitary odometers can be embedded in such cellular automata.

Definitions and background. Let $S=\left(s_{1}, s_{2}, \ldots\right)$ be a sequence of integers greater than 1. Define

$$
\mathbb{Z}(S):=\prod_{k \geq 1} \mathbb{Z} / s_{k} \mathbb{Z} \quad \text { and } \quad \widetilde{\mathbb{Z}}(S):=\operatorname{inv}_{k \rightarrow \infty} \lim \left(\mathbb{Z} / s_{1} \cdots s_{k} \mathbb{Z}, \beta_{k}\right),
$$

where the binding maps $\beta_{k}: s_{1} \cdots s_{k+1} \mathbb{Z} \rightarrow s_{1} \cdots s_{k} \mathbb{Z}$ are defined by

$$
z \mapsto z \bmod s_{1} \cdots s_{k} \text {. }
$$

Addition in $\mathbb{Z}(S)$ is "with carrying," addition in $\widetilde{\mathbb{Z}}(S)$ is coordinatewise, i.e. without carrying. $\mathbb{Z}(S)$ and $\widetilde{\mathbb{Z}}(S)$ are isomorphic, compact, abelian, topological groups [4].

The +1 map on $\mathbb{Z}(S)$ is defined by

$$
z \mapsto z+(1,0,0, \ldots)
$$

and the $+\tilde{1}$ map on $\widetilde{\mathbb{Z}}(S)$ is defined by

$$
z \mapsto z+(1,1, \ldots)
$$

$(\mathbb{Z}(S),+1)$ and $(\widetilde{\mathbb{Z}}(S),+\tilde{1})$ are topologically conjugate (any topological group isomorphism of $\mathbb{Z}(S)$ onto $\widetilde{\mathbb{Z}}(S)$ that takes 1 to $\tilde{1}$ is a topological conjugacy) and are called the $S$-adic odometer. When $S=(n, n, \ldots),(\mathbb{Z}(S),+1)$ is the well-known $n$-adic odometer, denoted $(\mathbb{Z}(n),+1)$.

By Theorem 7.6 of [2], a complete topological conjugacy invariant of $(\mathbb{Z}(S),+1)$ is the multiplicity function $\mathrm{MULT}_{S}:\{$ primes $\} \rightarrow\{0,1, \ldots, \infty\}$, defined by

$$
\operatorname{MULT}_{S}(p):=\sum_{i}\left\{\max j: p^{j} \text { divides } s_{i}\right\} .
$$

Thus $\operatorname{MULT}_{S}(p)$ is the total number of times that $p$ divides members of $S$.

Throughout this paper a two-sided cellular automaton $(X, T)$ will be a dynamical system defined on a closed, $T$-invariant subset of $A^{\mathbb{Z}}$, where $A$ is a finite alphabet and $T$ is given by a local rule $\tau: A^{2 m+1} \rightarrow A$ for some $m \geq 0$ as follows: $[T(x)]_{i}=\tau\left(x_{i-m}, \ldots, x_{i+m}\right)(i \in \mathbb{Z})$. We note that $T$ is continuous and commutes with the shift $\sigma: A^{\mathbb{Z}} \rightarrow A^{\mathbb{Z}}$, defined by $[\sigma(x)]_{i}=x_{i+1}(i \in \mathbb{Z})$. When appropriate, we will write $x \in A^{\mathbb{Z}}$ as $x_{L} \cdot x_{R}$, where the dot separates the negative indices from the non-negative ones. One-sided cellular automata are similarly defined.

When $A$ has $n$ elements, we may sometimes assume that $A=\mathbb{Z}_{n}$, the ring of integers modulo $n$. The cellular automaton defined on all doubly infinite sequences with entries from $\mathbb{Z}_{n}$ and local rule $x_{i} \mapsto x_{i}+x_{i+1} \bmod n(i \in \mathbb{Z})$ 
will be denoted $\left(\mathbb{Z}_{n}^{\mathbb{Z}}, T_{n}\right)$. The maps $T_{n}$ have no memory and so we define one-sided cellular automata $\left(T_{n}\right)_{R}: \mathbb{Z}_{n}^{\mathbb{N}_{0}} \rightarrow \mathbb{Z}_{n}^{\mathbb{N}_{0}}$ by the same local rule. Here $\mathbb{N}:=\{1,2, \ldots\}$ is the natural numbers and $\mathbb{N}_{0}:=\{0,1,2, \ldots\}$.

A more geometric class of cellular automata is the class of gliders-withreflecting-walls cellular automata [6, Example 6.5].

The alphabet for all these one-sided cellular automata is

$$
\{W, L, R, \emptyset\}
$$

where $W$ is a stationary wall, $L$ is a left-moving particle, $R$ is a right-moving particle, and $\emptyset$ is an empty space.

The spaces $X \subseteq A^{\mathbb{N}}$ satisfy: for every $x \in X, x_{1}=W, x_{i}=W$ for infinitely many $i$, and between any two consecutive $W$ there is exactly one particle.

The local rule for these automata is as follows:

- Walls do not move.

- If the space immediately to the left of $L$ is empty, then $L$ and $\emptyset$ change places. If the space immediately to the left of $L$ is $W$, then $L$ becomes $R$ but does not move.

- If the space immediately to the right of $R$ is empty, then $R$ and $\emptyset$ change places. If the space immediately to the right of $R$ is $W$, then $R$ becomes $L$ but does not move.

For a dynamical system $(X, f)$, where $X$ is a subset of some $A^{\mathbb{Z}}$ or $A^{\mathbb{N}}$, the space-time diagram of $(X, f)$ with seed $x$ is the array whose $(i, j)$ th entry is $\left[f^{j}(x)\right]_{i}$. It is a convenient way of visualizing the forward $f$-orbit of $x$, $\left\{f^{j}(x): j \geq 0\right\}$. Here we think of "increasing time" as going down. Spacetime diagrams for systems on one-sided sequences are similarly defined, and are convenient ways of visualizing odometers.

For dynamical systems $(X, f)$ and $(\widehat{X}, \widehat{f})$, we say that $(X, f)$ can be embedded in $(\widehat{X}, \widehat{f})$ if there is a closed, $\widehat{f}$-invariant subset $\widehat{X}^{\prime}$ of $\widehat{X}$ such that $(X, f)$ is topologically conjugate to $\left(\widehat{X}^{\prime}, \widehat{f} \widehat{X}_{\widehat{X}^{\prime}}\right)$.

Every odometer can be embedded in a gliders-with-reflectingwalls cellular automaton. Gliders-with-reflecting-walls cellular automata $(X, T)$ are defined on one-sided infinite sequences with entries from $\{W, L$, $R, \emptyset\}$, with local rules defined in the preceding section.

THEOREM 1. Every odometer can be embedded in a gliders-with-reflecting-walls cellular automaton.

Proof. Let $S=\left(s_{1}, s_{2}, \ldots\right)$.

First assume that at least one $s_{i}$ is even. Since the multiplicity function is a complete topological conjugacy invariant of $(\mathbb{Z}(S),+1)$, the order of the $s_{i}$ is irrelevant, so we may assume that $s_{1}$ is even. 
Consider the set $X$ of all points in $\{W, L, R, \emptyset\}^{\mathbb{N}}$ of the form

$$
W \leftarrow \frac{1}{2} s_{1} \rightarrow W \leftarrow \frac{1}{2} s_{1} s_{2} \rightarrow W \leftarrow \cdots,
$$

where the gaps contain exactly one particle. The columns of gaps in the space-time diagram of a gliders-with-reflecting-walls cellular automaton with any such point as seed are periodic with least periods $s_{1}, s_{1} s_{2}, \ldots$.

We show that this one-sided cellular automaton is topologically conjugate to $\left(\widetilde{\mathbb{Z}}\left(s_{1}, s_{2}, s_{3}, \ldots\right),+\widetilde{1}\right)$. Let $\widetilde{T}$ be the gliders-with-reflecting-walls cellular automaton map and label the gaps, left-to-right, $G_{1}, G_{2}, \ldots$ Consider the space-time diagram of $(X, \widetilde{T})$ with seed $\bar{x}$, defined by " $R$ appears at the extreme left of each gap." For $x$ in the forward $\widetilde{T}$-orbit-closure of $\bar{x}$, define

$$
x \mapsto z=\left(z_{1}, z_{2}, \ldots\right) \in \prod_{k \geq 1} \mathbb{Z} / s_{1} \cdots s_{k} \mathbb{Z}
$$

as follows. For $i \geq 1$, let $z_{i}, 0 \leq z_{i} \leq s_{1} \cdots s_{i}-1$, satisfy

$$
\left.x\right|_{G_{i}}=\widetilde{T}^{z_{i}}(\overleftarrow{R, \emptyset, \emptyset, \ldots, \emptyset)},
$$

$\longleftarrow \frac{1}{2} s_{1} s_{2} \cdots s_{i} \longrightarrow$

i.e. $(R, \emptyset, \emptyset, \ldots, \emptyset)$ appears in row $z_{i}$ in this space-time diagram. This map is continuous, one-to-one, and commutes with the appropriate actions, and so is a topological conjugacy.

Now assume that all the $s_{i}$ are odd. In this case the cellular automaton $\left(X, \widetilde{T}^{2}\right)$, defined on all points of $\{W, L, R, \emptyset\}^{\mathbb{N}}$ of the form

$$
W \leftarrow s_{1} \rightarrow W \leftarrow s_{1} s_{2} \rightarrow W \leftarrow \cdots,
$$

in the forward $\widetilde{T}^{2}$-orbit-closure of $\bar{x}$ (defined as above, $R$ at the extreme left of each gap), is topologically conjugate to $(\widetilde{\mathbb{Z}}(S),+\tilde{1})$.

An odometer can be embedded in a cellular automaton with local rule $x_{0}+x_{1}$ if and only if it is "finitary". The word "finitary" in the title of this section refers to odometers $(\mathbb{Z}(S),+1)$ such that the set of prime divisors of the members of $S$ is finite.

Throughout this section, $\left(\mathbb{Z}_{n}^{\mathbb{Z}}, T_{n}\right)$ will denote the two-sided cellular automaton with local rule $x_{i} \mapsto x_{i}+x_{i+1} \bmod n(i \in \mathbb{Z})$. To avoid notational clutter, we may write $T$ rather than $T_{n}$ when $n$ is clear.

Lemma 1. Let $\bar{x}=\ldots 000.100 \ldots$ and let $X$ be the forward $T_{n}$-orbitclosure of $\bar{x}$.

(1) For any $n \geq 2, \bar{x}_{R}:=100 \ldots$ is $\left(T_{n}\right)_{R}$-fixed.

(2) For any $x \in X$, if some column $\left[T_{n}^{j}(x)\right]_{i}(j \geq 0)$ in the space-time diagram of $\left(X, T_{n}\right)$ with seed $x$ is periodic with least period $m$, then the column immediately to the left, $\left[T_{n}^{j}(x)\right]_{i-1}(j \geq 0)$, is periodic with least period $m n^{\prime}$ for some factor $n^{\prime}$ of $n\left(n^{\prime}=1\right.$ or $n$ is possible). 
(3) For any $n \geq 2, \bar{x}$ has an infinite forward $T_{n}$-orbit.

(4) For $n=p$ prime, there exist $1=k_{1}<k_{2}<\cdots$ such that for every $i \geq 1$, the columns $\left[T_{p}^{j}(\bar{x})\right]_{i}(j \geq 0), i=-k_{i+1}+1, \ldots,-k_{i}$, are periodic with least period $p^{i}$.

Proof. Write $T$ in place of $T_{n}$. (1) is clear.

(2) We prove this part for $n=p$ prime, leaving it to the reader to supply the details for the general case. Suppose that column $i$ in the space-time diagram of $(X, T)$ with seed $x$ is periodic with least period $m:\left[T^{j}(x)\right]_{i}=$ $\left[T^{j+m}(x)\right]_{i}(j \geq 0)$. If

$$
\sum_{j=0}^{m-1}\left[T^{j}(x)\right]_{i} \equiv 0 \bmod p,
$$

then column $i-1$ is periodic with least period $m$. If

$$
\sum_{j=0}^{m-1}\left[T^{j}(x)\right]_{i} \not \equiv 0 \bmod p,
$$

then column $i-1$ is periodic with least period $p m$.

(3) If $\bar{x}$ has a finite forward $T$-orbit, then there exists $K \geq 0$ such that $x_{-k}=0$ for every point $x$ in this orbit and for every $k \geq K$. This contra$\operatorname{dicts}(2)$.

(4) follows from (1), (2), and (3).

We divide the "if and only if" statement of Theorem 2 into two separate theorems.

THEOREM 2A. Every finitary odometer $(\mathbb{Z}(S),+1)$, i.e. one such that the set of prime divisors of the members of $S$ is finite, can be embedded in the one-dimensional, two-sided cellular automaton $\left(\mathbb{Z}_{n}^{\mathbb{Z}}, T_{n}\right)$ with local rule

$$
x_{i} \mapsto x_{i}+x_{i+1} \bmod n \quad(i \in \mathbb{Z}),
$$

where $n$ is the product of the primes that divide infinitely many members of $S$.

Since the multiplicity function is a complete topological conjugacy invariant, every finitary odometer is topologically conjugate to one of the following two canonical forms:

(1) the $n$-adic odometer, $(\mathbb{Z}(n),+1):=(\mathbb{Z}(n, n, \ldots),+1)$, where $n$ is the product of distinct primes,

(2) $(\mathbb{Z}(m, n, n, \ldots),+1)$, where $m$ and $n$ are relatively prime and $n$ is the product of distinct primes.

Theorem 2A follows from Lemmas 2-7 below.

LEMMA 2. For $p$ prime and $m \geq 2$ such that $p$ is not a factor of $m$, both $(\mathbb{Z}(p),+1)$ and $(\mathbb{Z}(m, p, p, \ldots),+1)$ can be embedded in $\left(\mathbb{Z}_{p}^{\mathbb{Z}}, T_{p}\right)$. 
Proof. Throughout this proof, we write $T$ in place of $T_{p}$.

First we prove the lemma for $(\mathbb{Z}(p),+1)$. Consider the space-time diagram of $\left(\mathbb{Z}_{p}^{\mathbb{Z}}, T\right)$ with seed

$$
\bar{x}=\ldots 000.1000 \ldots
$$

We show that $T$ restricted to the forward $T$-orbit-closure of $\bar{x}$ is topologically conjugate to $(\mathbb{Z}(p),+1)$. Recall from Lemma $1(3),(1)$ that $\bar{x}$ has an infinite forward $T$-orbit and that $\bar{x}_{R}:=100 \ldots$ is $T_{R}$-fixed.

Define a mapping $x \mapsto z$ from the forward $T$-orbit-closure of $\bar{x}$ to $\mathbb{Z}(p)$ as follows. For $x$ in the forward $T$-orbit-closure of $\bar{x}$, let $z=\left(z_{1}, z_{2}, \ldots\right) \in \mathbb{Z}(p)$ be such that

$$
T^{\sum_{i=1}^{k} z_{i} p^{i-1}}(\bar{x}) \rightarrow x \quad \text { as } k \rightarrow \infty .
$$

That such a sequence exists follows from Lemma 1. (The partial sums of $\sum_{i=1}^{\infty} z_{i} p^{i-1}$ are the rows in the space-time diagram of $\left(\mathbb{Z}_{p}^{\mathbb{Z}}, T\right)$ with seed $\bar{x}$ at which the appropriate "right tails" of $x$ appear, so $z$ is well-defined.) This mapping is continuous, one-to-one, and commutes with the appropriate actions. Therefore it is a topological conjugacy.

The proof of the lemma for $(\mathbb{Z}(m, p, p, \ldots),+1)$ follows the proof for $(\mathbb{Z}(p),+1)$ provided we can find a seed $\bar{y}=\bar{y}_{L} \cdot \bar{y}_{R}$ such that $\bar{y}$ has an infinite forward $T$-orbit and $\bar{y}_{R}$ is $T_{R}$-periodic with least period $m$. That we can do this is Lemma 4 below.

LEMma 3. $\left(\mathbb{Z}_{p}^{\mathbb{N}_{0}}, T_{R}\right)$ is topologically conjugate to the full one-sided shift $\left(\mathbb{Z}_{p}^{\mathbb{N}_{0}}, \sigma_{L}\right)$, where $\sigma_{L}$ is the left-shift defined by $\left[\sigma_{L}(x)\right]_{i}:=x_{i+1}(i \geq 0)$.

Proof. The topological conjugacy $x \mapsto y$ is given by $y_{i}:=\left[T_{R}^{i}(x)\right]_{0}$ $(i \geq 0)$. For a more general result, see [1].

Lemma 4. Let $m \geq 1$. There is a point $\bar{y}=\bar{y}_{L} \cdot \bar{y}_{R}$ with an infinite forward $T$-orbit and such that $\bar{y}_{R}$ is $T_{R}$-periodic with least period $m$.

Proof. By Lemma 3 there is a $T_{R}$-periodic point $\bar{y}_{R}=\bar{y}_{0} \bar{y}_{1} \ldots$ with least period $m$. It follows from Lemma $1(2)$ that column 0 in the space-time diagram of $T$ (N.B. $T$, not $T_{R}$ ) with seed any left extension of $\bar{y}_{R}$ is periodic. So it suffices to show that $\bar{y}_{R}$ has a left extension such that the columns in the appropriate space-time diagram have arbitrarily large least periods.

By Lemma 3, for every $k \geq 1$ there are $p^{k} T_{R}^{k}$-fixed points. For $k=1$ (since $p^{2}>p^{1}$ ), there exist $\bar{y}_{-1}$ and $\bar{y}_{-2}$ such that $\bar{y}_{-2} \bar{y}_{-1} \bar{y}_{0} \ldots$ is not $T_{R^{-}}$ fixed. For $k=2$, there exist $\bar{y}_{-3}, \bar{y}_{-4}$, and $\bar{y}_{-5}$ such that $\bar{y}_{-5} \bar{y}_{-4} \ldots$ is not $T_{R}^{2}$-fixed. Continue with $k=3,4, \ldots$.

Lemma 5. If $(X, f)$ can be embedded in $(\widehat{X}, \widehat{f})$ and $(Y, g)$ can be embedded in $(\widehat{Y}, \widehat{g})$, then $(X, f) \times(Y, g)$ can be embedded in $(\widehat{X} \times \widehat{Y}, \widehat{f} \times \widehat{g})$. 
Lemma 6. Let $m, n \geq 2$ be relatively prime. Then $(\mathbb{Z}(m n),+1)$ is topologically conjugate to $(\mathbb{Z}(m) \times \mathbb{Z}(n),(+1,+1))$. If , in addition, $s \geq 2$ is relatively prime to both $m$ and $n$, then $(\mathbb{Z}(s, m n, m n, \ldots),+1)$ is topologically conjugate to $(\mathbb{Z}(s, m, m, \ldots) \times \mathbb{Z}(n),(+1,+1))$.

Proof. To prove the first statement it suffices to find a topological group isomorphism of $\mathbb{Z}(m n)$ onto $\mathbb{Z}(m) \times \mathbb{Z}(n)$ that takes $(1,0, \ldots) \in \mathbb{Z}(m n)$ to $((1,0, \ldots),(1,0, \ldots)) \in \mathbb{Z}(m) \times \mathbb{Z}(n)$.

Map $\mathbb{Z}(m n)$ to $\mathbb{Z}(m) \times \mathbb{Z}(n)$ by

$$
\left(z_{0}, z_{1}, \ldots\right) \mapsto\left(\left(z_{0}^{\prime}, z_{1}^{\prime}, \ldots\right),\left(z_{0}^{\prime \prime}, z_{1}^{\prime \prime}, \ldots\right)\right),
$$

where for every $k \geq 0, \sum_{i=0}^{k} z_{i}^{\prime} m^{i}$ is the beginning of the base $m$ expansion of $\sum_{i=0}^{k} z_{i}(m n)^{i}$; similarly for $z^{\prime \prime}$. This map is well-defined, takes $(1,0, \ldots)$ to $((1,0, \ldots),(1,0, \ldots))$, and satisfies all the conditions of topological group isomorphism, except possibly ontoness. To see that it maps $\mathbb{Z}(m n)$ onto $\mathbb{Z}(m) \times \mathbb{Z}(n)$, notice that it maps the set

$$
\{k(1,0, \ldots) \in \mathbb{Z}(m n): k \geq 0\},
$$

which is dense in $\mathbb{Z}(m n)$, onto the set

$$
\{(k(1,0, \ldots), k(1,0, \ldots)) \in \mathbb{Z}(m) \times \mathbb{Z}(n): k \geq 0\} .
$$

The latter set is dense in $\mathbb{Z}(m) \times \mathbb{Z}(n)$ because $m$ and $n$ are relatively prime.

The proof of the second statement is similar. We omit the details.

Lemma 7. Let $m, n \geq 2$ be relatively prime. Then $\left(\mathbb{Z}_{m n}^{\mathbb{Z}}, T_{m n}\right)$ is topologically conjugate to $\left(\mathbb{Z}_{m}^{\mathbb{Z}} \times \mathbb{Z}_{n}^{\mathbb{Z}}, T_{m} \times T_{n}\right)$.

Proof. Any ring isomorphism of $\mathbb{Z}_{m} \times \mathbb{Z}_{n}$ onto $\mathbb{Z}_{m n}$ is a topological conjugacy of $\left(\mathbb{Z}_{m}^{\mathbb{Z}} \times \mathbb{Z}_{n}^{\mathbb{Z}}, T_{m} \times T_{n}\right)$ onto $\left(\mathbb{Z}_{m n}^{\mathbb{Z}}, T_{m n}\right)$.

TheOREM 2B. If an odometer $(\mathbb{Z}(S),+1)$ can be embedded in the onedimensional, two-sided cellular automaton $\left(\mathbb{Z}_{n}^{\mathbb{Z}}, T_{n}\right)$ with local rule

$$
x_{i} \mapsto x_{i}+x_{i+1} \bmod n \quad(i \in \mathbb{Z}),
$$

then $(\mathbb{Z}(S),+1)$ is finitary, i.e. the set of prime divisors of the members of $S$ is finite.

Proof. Suppose that $(\mathbb{Z}(S),+1)$ is topologically conjugate to $\left(X, T_{n}\right)$, where $X$ is a closed, $T_{n}$-invariant subset of $\mathbb{Z}_{n}^{\mathbb{Z}}$. Consider the space-time diagram of $(\mathbb{Z}(S),+1)$ with seed $(0,0, \ldots)$. Every column is periodic and for $p$ prime, $p$ divides the least period of some column if and only if $p$ divides some $s \in S$.

It follows from the uniform continuity of the topological conjugacy and its inverse that every column in any space-time diagram of $\left(X, T_{n}\right)$ is periodic. For $p$ prime, $p$ divides the least period of some column in a space-time diagram of $\left(X, T_{n}\right)$ if and only if $p$ divides the least period of some column 
in the space-time diagram of $(\mathbb{Z}(S),+1)$ with seed $(0,0, \ldots)$. The proof is completed by applying the lemma below.

Lemma 8. For any $n \geq 2$, the set of primes $p$ such that $p$ divides the least period of some column in a space-time diagram of $\left(X, T_{n}\right)$ is finite.

Proof. Since every column in the space-time diagram of $(\mathbb{Z}(S),+1)$ with seed $(0,0, \ldots)$ is periodic, it follows from Lemma 1 that every column in any space-time diagram of $\left(X, T_{n}\right)$ is periodic. Furthermore, if column $i$ has least period $m$, then column $i-1$ has least period $n^{\prime} m$, where $n^{\prime}$ is a factor of $n$.

So if a column has least period $m$, then any prime that divides the least period of some column to its left also divides $m n$. Hence the set of all primes that divide the least period of any column is finite.

Acknowledgements. Some of this work was done while the first author was a guest of Trent University. He thanks it for its hospitality and support. The authors thank M. Pivato for introducing them to gliders with reflecting walls.

\section{References}

[1] F. Blanchard and A. Maass, Dynamical properties of one-sided cellular automata, Israel J. Math. 99 (1997), 149-174.

[2] J. Buescu and I. Stewart, Liapunov stability and adding machines, Ergodic Theory Dynam. Systems 15 (1995), 271-290.

[3] E. M. Coven, M. Pivato, and R. Yassawi, Prevalence of odometers in cellular automata, Proc. Amer. Math. Soc. 135 (2007), 815-821.

[4] T. Downarowicz, Survey of odometers and Toeplitz flows, in: Algebraic and Topological Dynamics, Contemp. Math. 385, Amer. Math. Soc., Providence, RI, 2005, 7-37.

[5] R. H. Herman, I. F. Putnam, and C. F. Skau, Ordered Bratteli diagrams, dimension groups and topological dynamics, Int. J. Math. 3 (1992), 827-864.

[6] J. Milnor, On the entropy geometry of cellular automata, Complex Systems 2 (1988), $357-385$.

[7] M. Pivato and R. Yassawi, Embedding Bratteli-Vershik systems in cellular automata, Ergodic Theory Dynam. Systems, to appear.

Ethan M. Coven

Department of Mathematics

Wesleyan University

Middletown, CT 06457-0128, U.S.A.

E-mail: ecoven@wesleyan.edu
Reem Yassawi

Department of Mathematics

Trent University

Peterborough, ON, Canada K9L 1Z8

E-mail: ryassawi@trentu.ca

Received 19 September 2008;

in revised form 16 April 2009 